\title{
A CHARATERIZATION OF COMMUTATORS FOR PARABOLIC SINGULAR INTEGRALS
}

\author{
YANPING CHEN and YONG DING*
}

\begin{abstract}
In this paper, the authors give a characterization of the $L^{p}$-boundedness of the commutators for the parabolic singular integrals. More precisely, the authors prove that if $b \in \mathrm{BMO}_{\varphi}\left(\mathrm{R}^{n}, \rho\right)$, then the commutator $[b, T]$ is a bounded operator from $L^{p}\left(\mathrm{R}^{n}\right)$ to the Orlicz space $L_{\psi}\left(\mathrm{R}^{n}\right)$, where the kernel function $\Omega$ has no any smoothness on the unit sphere $S^{n-1}$. Conversely, if assuming on $\Omega$ a slight smoothness on $S^{n-1}$, then the boundedness of $[b, T]$ from $L^{p}\left(\mathrm{R}^{n}\right)$ to $L_{\psi}\left(\mathrm{R}^{n}\right)$ implies that $b \in \mathrm{BMO}_{\varphi}\left(\mathrm{R}^{n}, \rho\right)$. The results in this paper improve essentially and extend some known conclusions.
\end{abstract}

\section{Introduction}

Suppose that $S^{n-1}=\left\{x \in \mathrm{R}^{n}:|x|=1\right\}$ is the unit sphere in $\mathrm{R}^{n}$ equipped with the Lebesgue measure $d \sigma$, where $|\cdot|$ denotes the Euclidean norm in $\mathrm{R}^{n}$. Let $b \in L_{\mathrm{loc}}\left(\mathrm{R}^{n}\right)$, then the commutator of the classical singular integral is defined by

$$
[b, T] f(x)=\text { p.v. } \int_{\mathrm{R}^{n}} \frac{\Omega(x-y)}{|x-y|^{n}}(b(x)-b(y)) f(y) d y,
$$

where $\Omega$ is homogeneous function of degree zero on $R^{n} \backslash\{0\}$, that is,

$$
\Omega(\mu x)=\Omega(x) \quad \text { for any } \quad \mu>0 \text { and } x \in \mathrm{R}^{n} \backslash\{0\} .
$$

Moreover, $\Omega \in L^{1}\left(S^{n-1}\right)$ satisfying the following cancellation condition:

$$
\int_{S^{n-1}} \Omega\left(x^{\prime}\right) d \sigma\left(x^{\prime}\right)=0 .
$$

In 1976, Coifman, Rochberg and Weiss gave the following result:

Theorem A ([2]). If $\Omega \in \operatorname{Lip}_{1}\left(S^{n-1}\right)$ satisfies (1.1) and (1.2), and $b \in$ BMO, then $[b, T]$ is bounded on $L^{p}\left(\mathrm{R}^{n}\right)(1<p<\infty)$. Conversely, if $\left[b, R_{j}\right]$

\footnotetext{
* This work was supported by NSF of China (Grants No. 10931001, 10901017) and SRFDPHE of China (Grant No. 20090003110018).

Received 17 October 2008.
} 
is bounded on $L^{p}\left(\mathrm{R}^{n}\right)$ for some $p, 1<p<\infty$ and all $j=1, \ldots, n$, then $b \in \mathrm{BMO}$, where $R_{j}$ is the $j$ 'th Riesz transform.

In 1978, Janson [6] extended Theorem A. To state the result in [6], we give some notations and definitions.

Let $\psi$ be a non-decreasing convex function on $\mathbf{R}_{+}$with $\psi(0)=0$, let $\psi^{-1}$ denote the inverse function of $\psi$. The Orlicz space $L_{\psi}\left(\mathrm{R}^{n}\right)$ is the set of functions $f$ satisfying

$$
\varrho(f, \psi)=\int_{\mathrm{R}^{n}} \psi(\lambda|f(y)|) d y<\infty
$$

for some $\lambda>0$. The norm in $L_{\psi}$ is defined by

$$
\|f\|_{L_{\psi}}=\inf _{\lambda>0} \frac{1}{\lambda}\left(1+\int_{\mathrm{R}^{n}} \psi(\lambda|f(y)|) d y\right)=\sup _{\substack{g \in L_{\psi^{*}} \\ \varrho\left(g, \psi^{*}\right) \leq 1}}\left|\int_{\mathrm{R}^{n}} f(y) g(y) d y\right|,
$$

where $\psi^{*}$ is the complementary Young function of $\psi$, which is given by

$$
\psi^{*}(s)=\sup _{0 \leq t<\infty}[s t-\psi(t)], \quad 0 \leq s<\infty .
$$

The following generalized Hölder's inequality holds in the Orlicz space $L_{\psi}\left(\mathrm{R}^{n}\right)$ (see [9] for its proof):

$$
\left|\int_{\mathrm{R}^{n}} f(y) g(y) d y\right| \leq\|f\|_{L_{\psi}}\|g\|_{L_{\psi^{*}}} .
$$

Now we give the definition of $\mathrm{BMO}_{\varphi}$ as follows. Denote

$$
M(b, Q)=\frac{1}{|Q|} \int_{Q}\left|b(x)-b_{Q}\right| d x,
$$

where $b_{Q}=\frac{1}{|Q|} \int_{Q} b(x) d x$ and $Q$ is a cube in $\mathrm{R}^{n}$. Let $\varphi$ be a positive function, then

$$
\mathrm{BMO}_{\varphi}\left(\mathrm{R}^{n}\right)=\left\{b \in L_{\mathrm{loc}}\left(\mathrm{R}^{n}\right):\|b\|_{\mathrm{BMO}_{\varphi}}=\sup _{\substack{x \in \mathrm{R}^{n} \\ r>0}} \frac{M(b, Q(x, r))}{\varphi(r)}<\infty\right\}
$$

where $Q(x, r)$ denotes the cube centered at $x$ and with diameter $r$.

With the above notations, Janson got the following conclusion:

Theorem B ([6]). Suppose that $\Omega \in C^{\infty}\left(S^{n-1}\right)$ satisfies (1.1) and (1.2) and $1<p<\infty$. Let $\varphi$ and $\psi$ be two non-decreasing positive functions on $\mathbf{R}_{+}$ 
connected by the relation $\varphi(r)=r^{n / p} \psi^{-1}\left(r^{-n}\right)$ (or equivalently $\psi^{-1}(t)=$ $\left.t^{1 / p} \varphi\left(t^{-1 / n}\right)\right)$. If $\psi$ is convex, $\psi(0)=0$ and $\psi(2 t) \leq C \psi(t)$, then $b$ belongs to $\mathrm{BMO}_{\varphi}$ if and only if $[b, T]$ maps $L^{p}\left(\mathrm{R}^{n}\right)$ boundedly into $L_{\psi}\left(\mathrm{R}^{n}\right)$.

Notice that in Theorem B, $\Omega$ was assumed on a very stronger smoothness condition on $S^{n-1}$. Hence, it is natural to ask if the conclusion of Theorem B holds still under a weaker condition of $\Omega$. In the paper, we will give a positive answer to this question. In fact, we will improve and extend Theorem B under the parabolic cases. Before stating our results, let us recall some definitions and some known results.

In 1966, Fabes and Rivière [5] introduced the parabolic singular integral. Let $\alpha_{1}, \ldots, \alpha_{n}$ be fixed real numbers with $\alpha_{i} \geq 1$. It is easy to see that for each fixed $x \in \mathrm{R}^{n}$, the function

$$
F(x, \rho)=\sum_{i=1}^{n} \frac{x_{i}^{2}}{\rho^{2 \alpha_{i}}}
$$

is a strictly decreasing function of $\rho>0$. Therefore, there exists an unique $\rho=\rho(x)$ such that $F(x, \rho)=1$. It was proved in [5] that $\rho$ is a metric on $\mathrm{R}^{n}$ and the metric space is denoted by $\left(\mathrm{R}^{n}, \rho\right)$. For $\mu>0$ and $x \in \mathrm{R}^{n}$, if we denote by

$$
\delta_{\mu}:\left(x_{1}, x_{2}, \ldots, x_{n}\right) \longrightarrow\left(\mu^{\alpha_{1}} x_{1}, \mu^{\alpha_{2}} x_{2}, \ldots, \mu^{\alpha_{n}} x_{n}\right)
$$

a dilation on $\mathrm{R}^{n}$, then we have the polar decomposition $x=\delta_{\rho} x^{\prime}$ with $x^{\prime} \in$ $S^{n-1}, \rho=\rho(x)$ and $d x=\rho^{\alpha-1} J\left(x^{\prime}\right) d \rho d \sigma\left(x^{\prime}\right)$, where

$$
J\left(x^{\prime}\right)=\alpha_{1} x_{1}^{\prime 2}+\cdots+\alpha_{n} x_{n}^{\prime 2} \quad \text { and } \quad \alpha=\sum_{i=1}^{n} \alpha_{i} .
$$

Suppose that $\Omega$ is a real valued and measurable function defined on $R^{n} \backslash\{0\}$. It is said that $\Omega$ is homogeneous of degree zero with respect to $\delta_{\mu}$, if for any $\mu>0$ and $x \in \mathrm{R}^{n} \backslash\{0\}$

$$
\Omega\left(\delta_{\mu} x\right)=\Omega(x) .
$$

Moreover, we also assume that $\Omega$ satisfies the following cancellation condition:

$$
\int_{S^{n-1}} \Omega\left(x^{\prime}\right) J\left(x^{\prime}\right) d \sigma\left(x^{\prime}\right)=0 .
$$

In [5], Fabes and Rivière proved that if $\Omega \in C^{1}\left(S^{n-1}\right)$ satisfying (1.4) and (1.5), then the parabolic singular integral operator $T$ is bounded on $L^{p}\left(\mathrm{R}^{n}\right)$ for 
$1<p<\infty$, where $T$ is defined by

$$
T f(x)=\text { p.v. } \int_{\mathrm{R}^{n}} \frac{\Omega(y)}{\rho(y)^{\alpha}} f(x-y) d y .
$$

Later, the above result was improved by Nagel, Riviere and Wainger [7] and the regularity condition assumed on $\Omega$ was removed.

Theorem C ([7]). If $\Omega \in L \log ^{+} L\left(S^{n-1}\right)$ satisfies (1.4) and (1.5), then the operator $T$ is bounded on $L^{p}\left(\mathrm{R}^{n}\right)$ for $1<p<\infty$.

Recently, the condition of $\Omega$ in Theorem $\mathrm{C}$ was weakened further by Chen, Ding and Fan:

Theorem D ([4]). If $\Omega \in H^{1}\left(S^{n-1}\right)$ satisfies (1.4) and (1.5), where $H^{1}\left(S^{n-1}\right)$ denotes the Hardy space on the unit sphere $S^{n-1}$, then the parabolic singular integral operator $T$ is bounded on $L^{p}\left(\mathrm{R}^{n}\right)$ for $1<p<\infty$.

Let us now turn to the definitions of the BMO space and the Lipschitz space on $\left(\mathrm{R}^{n}, \rho\right)$. Denote by $\mathscr{E}(x, r)=\{y: \rho(x-y)<r\}$ the ellipsoid centered at $x$ and with radius $r$. For $j>0, j \mathscr{E}$ denotes the $j$-times extension of the ellipsoid $\mathscr{E}$ with the same center. Moreover, $|\mathscr{E}(x, r)|$ is the Lebesgue measure of $\mathscr{E}(x, r)$, which is comparable to $r^{\alpha}$. Let $\mathscr{E}^{c}$ denote the complement of $\mathscr{E}$.

For a positive function $\varphi$, the parabolic $\mathrm{BMO}_{\varphi}\left(\mathrm{R}^{n}, \rho\right)$ space is defined by

$$
\mathrm{BMO}_{\varphi}\left(\mathrm{R}^{n}, \rho\right)=\left\{b \in L_{\mathrm{loc}}\left(\mathrm{R}^{n}\right):\|b\|_{\mathrm{BMO}_{\varphi}, \rho}=\sup _{\substack{x \in \mathrm{R}^{n} \\ r>0}} \frac{M(b, \mathscr{E}(x, r))}{\varphi(r)}<\infty\right\},
$$

where $M(b, \mathscr{E})=\frac{1}{|\mathscr{E}|} \int_{\mathscr{E}}\left|b(x)-b_{\mathscr{E}}\right| d x$, and $b_{\mathscr{E}}=\frac{1}{|\mathscr{E}|} \int_{\mathscr{E}} b(x) d x$. When $\varphi \equiv 1$, then we denote simply $\mathrm{BMO}_{1}\left(\mathrm{R}^{n}, \rho\right)$ by $\mathrm{BMO}\left(\mathrm{R}^{n}, \rho\right)$ and $\|b\|_{\mathrm{BMO}_{1}, \rho}$ by $\|b\|_{\mathrm{BMO}, \rho}$.

For $0<\beta \leq 1$, the definition of parabolic Lipschitz space $\Lambda_{\beta}\left(\mathrm{R}^{n}, \rho\right)$ is the following:

$$
\Lambda_{\beta}\left(\mathrm{R}^{n}, \rho\right)=\left\{b:\|b\|_{\Lambda_{\beta}, \rho}=\sup _{x, y \in \mathrm{R}^{n}} \frac{|b(x)-b(y)|}{\rho(x-y)^{\beta}}<\infty\right\} .
$$

For $b \in L_{\mathrm{loc}}\left(\mathrm{R}^{n}\right)$, the commutator $[b, T]$ of parabolic singular integral operator is defined by

$$
[b, T] f(x)=\text { p.v. } \int_{\mathrm{R}^{n}} \frac{\Omega(x-y)}{\rho(x-y)^{\alpha}}(b(x)-b(y)) f(y) d y .
$$

The commutator of the parabolic singular integral operator arises naturally in the theory of parabolic PDE. In 1996, Bramanti and Cerutti [1] proved the 
commutator of parabolic singular integral operator is bounded on $L^{p}\left(\mathrm{R}^{n}\right)$ and gave some applications of the commutator in the theory of parabolic PDE.

TheOREM E ([1]). Suppose that $b \in \operatorname{BMO}\left(\mathrm{R}^{n}, \rho\right)$ and that $\Omega \in \operatorname{Lip}_{\beta}\left(S^{n-1}\right)$ $(0<\beta \leq 1)$ satisfies (1.4) and (1.5), then $[b, T]$ is bounded on $L^{p}\left(\mathrm{R}^{n}\right)$ $(1<p<\infty)$.

In 2004, Palagachev and Softova [8] gave the boundedness of commutator of parabolic singular integral with variable kernel on the Morrey space and gave some applications of the commutators in studying parabolic PDE.

Recently, we improved Theorem E and removed the regularity condition assumed on $\Omega$ in Theorem $\mathrm{E}$.

Theorem F ([3]). Suppose $\Omega \in L\left(\log ^{+} L\right)^{2}\left(S^{n-1}\right)$ satisfies (1.4) and (1.5). If $b \in \mathrm{BMO}\left(\mathrm{R}^{n}, \rho\right)$, then the commutator $[b, T]$ defined in (1.7) is bounded on $L^{p}\left(\mathrm{R}^{n}\right)$ for $1<p<\infty$.

The main results in this paper are as follows.

THEOREM 1.1. Let $\psi$ be a non-decreasing convex function on $\mathrm{R}_{+}$with $\psi(0)=0$ and $\psi(2 t) \leq C \psi(t)$. For $1<p<\infty$, denote $\varphi(r)=r^{\alpha / p} \psi^{-1}\left(r^{-\alpha}\right)$ (or equivalently, $\psi^{-1}(t)=t^{1 / p} \varphi\left(t^{-1 / \alpha}\right)$ ). Suppose that $0<\beta \leq 1$ satisfies $1<p<\alpha / \beta$ and $\Omega \in L^{\frac{\alpha}{\alpha-\beta}}\left(S^{n-1}\right)$ satisfying (1.4) and (1.5). Then for $b \in \mathrm{BMO}_{\varphi}\left(\mathrm{R}^{n}, \rho\right),[b, T]$ is bounded operator from $L^{p}\left(\mathrm{R}^{n}\right)$ to $L_{\psi}\left(\mathrm{R}^{n}\right)$.

The following result can be seen a reverse of Theorem 1.1.

TheOREM 1.2. Suppose that $\Omega$ satisfies (1.4) and (1.5) and there are constants $C_{1}>0$ and $\gamma>1$ such that for any $x^{\prime}, y^{\prime} \in S^{n-1}$

$$
\left|\Omega\left(x^{\prime}\right)-\Omega\left(y^{\prime}\right)\right| \leq \frac{C_{1}}{\left(\log \frac{2}{\rho\left(x^{\prime}-y^{\prime}\right)}\right)^{\gamma}} .
$$

If $[b, T]$ for some $1<p<\infty$ maps $L^{p}\left(\mathrm{R}^{n}\right)$ boundedly into $L_{\psi}\left(\mathrm{R}^{n}\right)$, then $b \in \mathrm{BMO}_{\varphi}\left(\mathrm{R}^{n}, \rho\right)$, where $\psi$ and $\varphi$ are both as in Theorem 1.1.

In particular, by taking $\varphi(r) \equiv 1$ and $\psi(t)=t^{p}$ for $1<p<\infty$ in Theorem 1.1 and Theorem 1.2, we get the following corollary of Theorem 1.1 and Theorem 1.2:

COROLlary 1.3. If $\Omega$ satisfies (1.4), (1.5) and (1.8), then the commutator $[b, T]$ is a bounded operator on $L^{p}\left(\mathrm{R}^{n}\right)(1<p<\infty)$ if and only if $b \in$ $\operatorname{BMO}\left(\mathrm{R}^{n}, \rho\right)$.

For $1<p<q<\infty$, if we take $\psi(t)=t^{q}$, then $\varphi(r)=r^{\alpha / p} r^{-\alpha / q}$ in Theorem 1.1 and Theorem 1.2. Notice that $\mathrm{BMO}_{t^{\beta}}\left(\mathrm{R}^{n}, \rho\right)=\Lambda_{\beta}\left(\mathrm{R}^{n}, \rho\right)$ (see 
Lemma 3.4 in Section 3), we therefore get another corollary of Theorem 1.1 and Theorem 1.2:

Corollary 1.4. Suppose that $1<p<q<\infty$ with $1 / p-1 / q \leq 1 / \alpha$. If $\Omega$ satisfies (1.4), (1.5) and (1.8), then $[b, T]$ is a bounded operator from $L^{p}\left(\mathrm{R}^{n}\right)$ to $L^{q}\left(\mathrm{R}^{n}\right)$ if and only if $b \in \Lambda_{\alpha\left(\frac{1}{p}-\frac{1}{q}\right)}\left(\mathrm{R}^{n}, \rho\right)$.

REMARK 1.5. Notice that the regularity condition assumed on $\Omega$ has been removed in Theorem 1.1, hence the conclusion of Theorem 1.1 is a substantial improvement of Theorem B and Theorem E. Moreover, it is easy to see that the condition (1.8) is weaker than the Lipshitz condition $\operatorname{Lip}_{\beta}\left(S^{n-1}\right)(0<\beta \leq 1)$. So Theorem 1.2 is also a substantial improvement and extension of Theorem B and Uchiyama's result in [11].

In the proof of Theorem 1.1, we will need to apply the $\left(L^{p}, L^{q}\right)$-boundedness of the parabolic fractional integral $T_{\Omega, \beta}$, which is an extension of the Hardy-Littlewood-Sobolev theorem for the Riesz potential (see [10]). So, these results have also itself independent interest. Here the parabolic fractional integral is defined by

$$
T_{\Omega, \beta} f(x)=\int_{\mathrm{R}^{n}} \frac{\Omega(y)}{\rho(y)^{\alpha-\beta}} f(x-y) d y,
$$

where $0<\beta<\alpha$ with $\Omega \in L^{\frac{\alpha}{\alpha-\beta}}\left(S^{n-1}\right)$ satisfying (1.4).

THeOREm 1.6. Suppose that $0<\beta<\alpha$, and that $\Omega \in L^{\frac{\alpha}{\alpha-\beta}}\left(S^{n-1}\right)$ satisfies (1.4).

(i) $T_{\Omega, \beta}$ is of weak type $\left(1, \frac{\alpha}{\alpha-\beta}\right)$. That is, there exists a constant $C>0$ such that for any $f \in L^{1}\left(\mathrm{R}^{n}\right)$ and $\lambda>0$,

$$
\left|\left\{x \in \mathrm{R}^{n}:\left|T_{\Omega, \beta} f(x)\right|>\lambda\right\}\right| \leq\left(\frac{C}{\lambda}\|f\|_{1}\right)^{\frac{\alpha}{\alpha-\beta}} .
$$

(ii) For $1<p<\frac{\alpha}{\beta}$ and $\frac{1}{q}=\frac{1}{p}-\frac{\beta}{\alpha}, T_{\Omega, \beta}$ is of type $(p, q)$.

Corollary 1.7. Suppose that $\Omega \in L^{\frac{\alpha}{\alpha-\beta}}\left(S^{n-1}\right)(0<\beta \leq 1)$ satisfies (1.4) and (1.5), let $b \in \Lambda_{\beta}\left(\mathrm{R}^{n}, \rho\right)$. Then the commutator $[b, T]$ defined in (1.7) is a bounded operator from $L^{p}\left(\mathrm{R}^{n}\right)$ to $L^{q}\left(\mathrm{R}^{n}\right)$ for $1<p<\alpha / \beta$ and $1 / q=1 / p-\beta / \alpha$. More precisely, we have $\|[b, T] f\|_{q} \leq C\|b\|_{\Lambda_{\beta}, \rho}\|f\|_{p}$, where the constant $C$ is independent of $b$ and $f$. 


\section{Proofs of Theorem 1.1 and Theorem 1.6}

Let us begin with the proof of Theorem 1.6.

Proof OF TheOREm 1.6. First we prove that for $1 \leq p<\frac{\alpha}{\beta}$ and $\frac{1}{q}=\frac{1}{p}-\frac{\beta}{\alpha}$, $T_{\Omega, \beta}$ is of weak type $(p, q)$. Set $K(x)=\frac{\Omega(x)}{\rho(x)^{\alpha-\beta}}$ and $E(s)=\left\{x \in \mathrm{R}^{n}\right.$ : $|K(x)|>s\}$ for $s>0$. Then

$$
|E(s)| \leq A s^{-\frac{\alpha}{\alpha-\beta}},
$$

where $A$ depends only on $\alpha, \beta$ and $\Omega$. In fact, by (1.4) and $\left|J\left(x^{\prime}\right)\right| \leq \alpha$, we have that

$$
\begin{aligned}
|E(s)| & \leq \frac{1}{s} \int_{E(s)} \frac{|\Omega(x)|}{\rho(x)^{\alpha-\beta}} d x \\
& \leq \frac{1}{s} \int_{S^{n-1}}\left|J\left(x^{\prime}\right)\right|\left|\Omega\left(x^{\prime}\right)\right| \int_{0}^{\left(\frac{\left|\Omega\left(x^{\prime}\right)\right|}{s}\right)^{\frac{1}{\alpha-\beta}}} r^{\beta-1} d r d \sigma\left(x^{\prime}\right) \\
& \leq A s^{-\frac{\alpha}{\alpha-\beta}} .
\end{aligned}
$$

Obviously, we may take $A=\frac{\alpha}{\beta}\|\Omega\|_{L^{\frac{\alpha}{\alpha-\beta}\left(S^{n-1}\right)}}^{\frac{\alpha}{\alpha-\beta}}$. Now for fixed $\mu>0$, let

$$
K_{1}(x)=\operatorname{sgn}(K(x))(|K(x)|-\mu) \chi_{E(\mu)}(x)
$$

and $K_{2}(x)=K(x)-K_{1}(x)$. It is easy to see that $\left\|K_{2}\right\|_{\infty} \leq \mu$. Thus for $1<p<\frac{\alpha}{\beta}$, from (2.1) we get

$$
\begin{aligned}
\int_{\mathrm{R}^{n}}\left|K_{2}(x)\right|^{p^{\prime}} d x & =p^{\prime} \int_{0}^{\mu} s^{p^{\prime}-1}|E(s)| d s \leq p^{\prime} A \int_{0}^{\mu} s^{p^{\prime}-1-\frac{\alpha}{\alpha-\beta}} d s \\
& =\frac{\alpha-\beta}{\alpha} A q \mu^{\frac{\alpha}{\alpha-\beta} \frac{p^{\prime}}{q}} .
\end{aligned}
$$

Hence, when $1 \leq p<\frac{\alpha}{\beta}$, we obtain that

$$
\left\|K_{2}\right\|_{p^{\prime}} \leq\left(\frac{\alpha-\beta}{\alpha} A q\right)^{\frac{1}{p^{\prime}}} \mu^{\frac{\alpha}{(\alpha-\beta) q}} .
$$

So, for $f \in L^{p}\left(\mathrm{R}^{n}\right)$, Hölder's inequality implies that

$$
\left\|K_{2} * f\right\|_{\infty} \leq\left(\frac{\alpha-\beta}{\alpha} A q\right)^{\frac{1}{p^{\prime}}} \mu^{\frac{\alpha}{(\alpha-\beta) q}}\|f\|_{p} .
$$


For any $\lambda>0$, set $\mu$ to satisfy

$$
\left(\frac{\alpha-\beta}{\alpha} A q\right)^{\frac{1}{p^{\prime}}} \mu^{\frac{\alpha}{(\alpha-\beta) q}}\|f\|_{p}=\frac{\lambda}{2}
$$

Then

$$
\left|\left\{x \in \mathrm{R}^{n}:\left|K_{2} * f(x)\right|>\frac{\lambda}{2}\right\}\right|=0 .
$$

Thus

$$
\begin{aligned}
\left|\left\{x \in \mathrm{R}^{n}:\left|T_{\Omega, \beta} f(x)\right|>\lambda\right\}\right| & \leq\left|\left\{x \in \mathrm{R}^{n}:\left|K_{1} * f(x)\right|>\frac{\lambda}{2}\right\}\right| \\
& \leq\left(\frac{2}{\lambda}\left\|K_{1} * f\right\|_{p}\right)^{p} .
\end{aligned}
$$

It follows from (2.1) that

$$
\begin{aligned}
\int_{\mathrm{R}^{n}}\left|K_{1}(x)\right| d x & =\int_{E(\mu)}(|K(x)|-\mu) d x \leq \int_{0}^{\infty}|E(t+\mu)| d t \\
& \leq A \int_{\mu}^{\infty} t^{-\frac{\alpha}{\alpha-\beta}} d t=\frac{\beta A}{\alpha-\beta} \mu^{-\frac{\beta}{\alpha-\beta}}
\end{aligned}
$$

For any $f \in L^{\infty}\left(\mathrm{R}^{n}\right)$ and $x \in \mathrm{R}^{n}$, by (2.4), we conclude that

$$
\left|K_{1} * f(x)\right| \leq\|f\|_{\infty} \int_{\mathrm{R}^{n}}\left|K_{1}(x)\right| d x \leq \frac{\beta A}{\alpha-\beta} \mu^{-\frac{\beta}{\alpha-\beta}}\|f\|_{\infty} .
$$

For any $f \in L^{1}\left(\mathrm{R}^{n}\right)$, it also implies that

(2.6) $\left\|K_{1} * f\right\|_{1} \leq \int_{\mathrm{R}^{n}} \int_{\mathrm{R}^{n}}\left|K_{1}(x-y)\left\|f(y) \mid d y d x \leq \frac{\beta A}{\alpha-\beta} \mu^{-\frac{\beta}{\alpha-\beta}}\right\| f \|_{1}\right.$.

Thus (2.5) and (2.6) show that $T_{1}: f \rightarrow K_{1} * f$ is of type $(\infty, \infty)$ and of type $(1,1)$. The Riesz-Thörin theorem leads to that $T_{1}$ is also of $(p, p)$ type for $1<p<\infty$, and

$$
\left\|T_{1}\right\|_{(p, p)} \leq \frac{\beta A}{\alpha-\beta} \mu^{-\frac{\beta}{\alpha-\beta}} .
$$

Combining (2.3) with (2.7) yields that (2.8)

$$
\left|\left\{x \in \mathrm{R}^{n}:\left|T_{\Omega, \beta} f(x)\right|>\lambda\right\}\right| \leq\left(\frac{2}{\lambda} \frac{\beta A}{\alpha-\beta} \mu^{-\frac{\beta}{\alpha-\beta}}\|f\|_{p}\right)^{p}=C\left(\frac{1}{\lambda}\|f\|_{p}\right)^{q} .
$$


where $C$ is independent of $\lambda$ and $f$.

Therefore, (2.8) tells us that $T_{\Omega, \beta}$ is of weak type $(p, q)$ for $1 \leq p<\frac{\alpha}{\beta}$ and $\frac{1}{q}=\frac{1}{p}-\frac{\beta}{\alpha}$. For $1<p<\frac{\alpha}{\beta}$, take $p_{0}$ such that $p<p_{0}<\frac{\alpha}{\beta}$ and let $q_{0}$ satisfy $1 / q_{0}=1 / p_{0}-\beta / \alpha$. By (2.8) and applying Marcinkiewicz interpolation theorem, we obtain that $T_{\Omega, \beta}$ is of type $(p, q)$, where $1 / q=1 / p-\beta / \alpha$. Thus we complete the proof of Theorem 1.6.

Proof of Corollary 1.7. For any $f \in L^{p}\left(\mathrm{R}^{n}\right)$ and $x \in \mathrm{R}^{n}$, by the definition of the parabolic fractional integral $T_{\Omega, \beta}$, we have the following pointwise relationship between the commutator $[b, T](f)(x)$ and $T_{|\Omega|, \beta}(|f|)(x)$ :

$$
\begin{aligned}
|[b, T](f)(x)| & \leq \int_{\mathrm{R}^{n}}|b(x)-b(y)| \frac{|\Omega(x-y)|}{\rho(x-y)^{\alpha}}|f(y)| d y \\
& \leq C\|b\|_{\Lambda_{\beta}, \rho} \int_{\mathrm{R}^{n}} \frac{|\Omega(x-y)|}{\rho(x-y)^{\alpha-\beta}}|f(y)| d y \\
& =C\|b\|_{\Lambda_{\beta}, \rho} T_{|\Omega|, \beta}(|f|)(x) .
\end{aligned}
$$

Then by Theorem 1.6 we get

$$
\|[b, T](f)\|_{q} \leq C\|b\|_{\Lambda_{\beta}, \rho}\left\|T_{|\Omega|, \beta}(|f|)\right\|_{q} \leq C\|b\|_{\Lambda_{\beta}, \rho}\|f\|_{p} .
$$

Before proving Theorem 1.1, let us give two lemmas. Suppose that $\eta \in$ $C_{0}^{\infty}\left(\mathrm{R}^{n}\right)$ with $\operatorname{supp}(\eta) \subset\{x: \rho(x)<1\}$ and $\int_{\mathrm{R}^{n}} \eta(y) d y=1$. For $r>0$ and $b \in \mathrm{BMO}_{\varphi}\left(\mathrm{R}^{n}, \rho\right)$, we denote

$$
b_{r}(x)=\int_{\mathrm{R}^{n}} b\left(x-\delta_{r} y\right) \eta(y) d y .
$$

Lemma 2.1 ([6]). Suppose that $b \in \mathrm{BMO}_{\varphi}\left(\mathrm{R}^{n}, \rho\right)$ with $\|b\|_{\mathrm{BMO}_{\varphi}, \rho} \leq 1$. Then for any $r>0, b-b_{r} \in \mathrm{BMO}_{\varphi}\left(\mathrm{R}^{n}, \rho\right)$ and $\left\|b-b_{r}\right\|_{\mathrm{BMO}_{\varphi, \rho}} \leq C \varphi(r)$.

LeMma 2.2. Let $0<\beta \leq 1$. If $b \in \mathrm{BMO}_{\varphi}\left(\mathrm{R}^{n}, \rho\right)$, then $b_{r} \in \Lambda_{\beta}\left(\mathrm{R}^{n}, \rho\right)$ and

$$
\left\|b_{r}\right\|_{\Lambda_{\beta}, \rho} \leq C r^{-\beta} \varphi(r)\|b\|_{\mathrm{BMO}_{\varphi}, \rho} .
$$

Proof. By the definition (2.9) of $b_{r}$, we get

$$
\begin{aligned}
& \mid b_{r}(x)- b_{r}(y) \mid \\
& \quad=\left|r^{-\alpha} \int_{\mathrm{R}^{n}} b(z) \eta\left(\delta_{r^{-1}}(x-z)\right) d z-r^{-\alpha} \int_{\mathrm{R}^{n}} b(z) \eta\left(\delta_{r^{-1}}(y-z)\right) d z\right| .
\end{aligned}
$$

Note that

$$
r^{-\alpha} \int_{\mathrm{R}^{n}} \eta\left(\delta_{r^{-1}} y\right) d y=\int_{\mathrm{R}^{n}} \eta(y) d y=1,
$$


for any $v$, independent of $z$, we have

$$
\begin{aligned}
& \left|b_{r}(x)-b_{r}(y)\right| \\
& \quad=r^{-\alpha}\left|\int_{\mathrm{R}^{n}}(b(z)-v) \eta\left(\delta_{r^{-1}}(x-z)\right) d z-\int_{\mathrm{R}^{n}}(b(z)-v) \eta\left(\delta_{r^{-1}}(y-z)\right) d z\right| \\
& \quad \leq r^{-\alpha} \int_{\mathrm{R}^{n}}\left|b(z)-v \| \eta\left(\delta_{r^{-1}}(x-z)\right)-\eta\left(\delta_{r^{-1}}(y-z)\right)\right| d z .
\end{aligned}
$$

If $\frac{\rho(x-y)}{r} \leq 1$, by $\|\eta\|_{\infty} \leq C$, we get

$$
\begin{aligned}
& \left|b_{r}(x)-b_{r}(y)\right| \\
& \quad \leq C r^{-\alpha} \int_{\mathrm{R}^{n}}|b(z)-v|\left|\eta\left(\delta_{r^{-1}}(x-z)\right)-\eta\left(\delta_{r^{-1}}(y-z)\right)\right|^{\beta} d z \\
& \quad \leq C r^{-\alpha}\left|\delta_{r^{-1}}(x-y)\right|^{\beta} \int_{\mathrm{R}^{n}}|b(z)-v|\left|\nabla \eta\left(\delta_{r^{-1}}(\iota x+(1-\iota) y-z)\right)\right|^{\beta} d z
\end{aligned}
$$

where $0<\iota<1$. Let $\mathscr{E}=\{z: \rho(\iota x+(1-\iota) y-z)<r\}$ and $v=b_{\mathscr{E}}$. Then by $\left|(x-y)_{i}\right| \leq \rho(x-y)^{\alpha_{i}}, \operatorname{supp}(\nabla \eta) \subset\{x: \rho(x)<1\}$ and $\|\nabla \eta\|_{\infty} \leq C$, we have

$$
\begin{aligned}
& \left|b_{r}(x)-b_{r}(y)\right| \\
& \quad \leq C\left|\delta_{r^{-1}}(x-y)\right|^{\beta} r^{-\alpha} \int_{\mathscr{E}}\left|b(z)-b_{\mathscr{E}}\right| d z \\
& \quad \leq C \varphi(r)\|b\|_{\mathrm{BMO}_{\varphi}, \rho}\left(r^{-2 \alpha_{1}}\left(x_{1}-y_{1}\right)^{2}+\cdots+r^{-2 \alpha_{n}}\left(x_{n}-y_{n}\right)^{2}\right)^{\beta / 2} \\
& \quad \leq C \varphi(r)\|b\|_{\mathrm{BMO}_{\varphi}, \rho}\left(\left(\frac{\rho(x-y)}{r}\right)^{2 \alpha_{1}}+\cdots+\left(\frac{\rho(x-y)}{r}\right)^{2 \alpha_{n}}\right)^{\beta / 2} \\
& \quad \leq C \varphi(r)\|b\|_{\mathrm{BMO}_{\varphi}, \rho}\left(\frac{\rho(x-y)}{r}\right)^{\beta} .
\end{aligned}
$$

If $\frac{\rho(x-y)}{r} \geq 1$, we get

$$
\begin{aligned}
&\left|b_{r}(x)-b_{r}(y)\right| \\
& \leq C r^{-\alpha} \int_{\mathrm{R}^{n}}|b(z)-v|\left|\eta\left(\delta_{r^{-1}}(x-z)\right)-\eta\left(\delta_{r^{-1}}(y-z)\right)\right|^{\beta / \max \left\{\alpha_{i}\right\}} d z \\
& \leq C r^{-\alpha}\left|\delta_{r^{-1}}(x-y)\right|^{\beta / \max \left\{\alpha_{i}\right\}} \int_{\mathrm{R}^{n}}|b(z)-a| \\
& \quad \times\left|\nabla \eta\left(\delta_{r^{-1}}(\iota x+(1-\iota) y-z)\right)\right|^{\beta / \max \left\{\alpha_{i}\right\}} d z .
\end{aligned}
$$


Let $\mathscr{E}=\{z: \rho(\iota x+(1-\iota) y-z)<r\}$ and $v=b_{\mathscr{E}}$, then by $\left|(x-y)_{i}\right| \leq$ $\rho(x-y)^{\alpha_{i}}, \operatorname{supp}(\nabla \eta) \subset\{x: \rho(x)<1\}$, and $\|\nabla \eta\|_{\infty} \leq C$, we get

$$
\begin{aligned}
& \left|b_{r}(x)-b_{r}(y)\right| \\
& \quad \leq C\left|\delta_{r^{-1}}(x-y)\right|^{\beta / \max \left\{\alpha_{i}\right\}} r^{-\alpha} \int_{\mathscr{E}}\left|b(z)-b_{\mathscr{E}}\right| d z \\
& \leq C \varphi(r)\|b\|_{\mathrm{BMO}_{\varphi}, \rho}\left(\left(\frac{\rho(x-y)}{r}\right)^{2 \alpha_{1}}+\cdots+\left(\frac{\rho(x-y)}{r}\right)^{2 \alpha_{n}}\right)^{\frac{\beta}{2 \max \left\{\alpha_{i}\right\}}} \\
& \quad \leq C \varphi(r)\|b\|_{\mathrm{BMO}_{\varphi}, \rho}\left(\frac{\rho(x-y)}{r}\right)^{\beta} .
\end{aligned}
$$

Thus (2.10) follows from the above estimates in two cases. i.e.,

For a measurable function $f$, denote by $m_{f}$ the distribution function of $f$,

$$
m_{f}(t)=|\{x:|f(x)|>t\}| \quad \text { for } t>0 .
$$

Lemma 2.3 ([6]). Suppose that $1 \leq p_{2}<p<p_{1}<\infty, \theta$ is a nonincreasing function on $\mathbf{R}_{+}, L$ is a linear operator such that

$$
\begin{cases}m_{L g}\left(t^{1 / p_{1}} \cdot \theta(t)\right) \leq \frac{C}{t}, & \text { if }\|g\|_{p_{1}} \leq 1, \\ m_{L g}\left(t^{1 / p_{2}} \cdot \theta(t)\right) \leq \frac{C}{t}, & \text { if }\|g\|_{p_{2}} \leq 1 .\end{cases}
$$

Then

$$
\int_{0}^{\infty} m_{L g}\left(2 t^{1 / p} \cdot \theta(t)\right) d t \leq C \quad \text { if } \quad\|g\|_{p} \leq\left(p / p_{1}\right)^{1 / p} .
$$

Proof OF TheOrem 1.1. We will apply some idea taken from [6] to prove Theorem 1.1. We may assume that $\|b\|_{\mathrm{BMO}_{\varphi}, \rho} \leq 1$, then by (2.10) $\left\|b_{r}\right\|_{\Lambda_{\beta}, \rho} \leq$ $\mathrm{Cr}^{-\beta} \varphi(r)$ for $r>0$. Choose $p_{i}(i=1,2)$ such that $1<p_{2}<p<p_{1}<\frac{\alpha}{\beta}$ and denote $1 / q_{i}=1 / p_{i}-\beta / \alpha$. Then by Corollary 1.7, for $f \in L^{p_{i}}(i=1,2)$ with $\|f\|_{p_{i}} \leq 1$

$$
\left\|\left[b_{r}, T\right] f\right\|_{q_{i}} \leq C r^{-\beta} \varphi(r) .
$$

On the other hand, by Lemmas 2.1 we know that $b-b_{r} \in \mathrm{BMO}_{\varphi}\left(\mathrm{R}^{n}, \rho\right)$ with $\left\|b-b_{r}\right\|_{\mathrm{BMO}_{\varphi, \rho}} \leq C \varphi(r)$. Note that $\Omega \in L^{\frac{\alpha}{\alpha-\beta}}\left(S^{n-1}\right) \subset L\left(\log ^{+} L\right)^{2}\left(S^{n-1}\right)$, applying Theorem $\mathrm{F}$ for $i=1,2$ and $f \in L^{p_{i}}$ with $\|f\|_{p_{i}} \leq 1$, we have

$$
\left\|\left[b-b_{r}, T\right] f\right\|_{p_{i}} \leq C \varphi(r) .
$$


We now take $r=t^{-1 / \alpha}$, by (2.12) and (2.13) we obtain the following weak estimate:

$$
m_{[b, T] f}\left(t^{1 / p_{i}} \varphi\left(t^{-1 / \alpha}\right)\right) \leq\left(\frac{2 C \varphi(r)}{t^{1 / p_{i}} \varphi(r)}\right)^{p_{i}}+\left(\frac{2 C r^{-\beta} \varphi(r)}{t^{1 / p_{i}} \varphi(r)}\right)^{q_{i}}=\frac{C}{t} .
$$

If set $\theta(t)=\varphi\left(t^{-1 / \alpha}\right)$, then (2.14) is just (2.11). Hence, by Lemma 2.3, when $\|f\|_{p} \leq\left(p / p_{1}\right)^{1 / p}$ we have

$$
\int_{\mathrm{R}^{n}} \psi\left(\frac{|[b, T] f(x)|}{2}\right) d x=\int_{0}^{\infty} m_{[b, T] f}\left(2 \psi^{-1}(t)\right) d t \leq C .
$$

This shows that $\|[b, T] f\|_{L_{\psi}} \leq C$ and Theorem 1.1 is proved.

\section{Some lemmas}

In this section, we give some lemmas which will be used in the proof of Theorem 1.2.

Lemma 3.1. If $\rho(x) \geq 4 \rho(y)$, then

$$
\rho\left((x-y)^{\prime}-x^{\prime}\right) \leq 3 \frac{\rho(y)}{\rho(x)}
$$

where $x^{\prime}=\left(\frac{x_{1}}{\rho(x)^{\alpha_{1}}}, \ldots, \frac{x_{n}}{\rho(x)^{\alpha_{n}}}\right) \in S^{n-1}$.

Proof. Write

$$
\rho\left((x-y)^{\prime}-x^{\prime}\right)=\rho\left(\frac{x_{1}-y_{1}}{\rho(x-y)^{\alpha_{1}}}-\frac{x_{1}}{\rho(x)^{\alpha_{1}}}, \ldots, \frac{x_{n}-y_{n}}{\rho(x-y)^{\alpha_{n}}}-\frac{x_{n}}{\rho(x)^{\alpha_{n}}}\right) .
$$

Denote $\varphi_{i}(x)=\frac{x_{i}}{\rho(x)^{\alpha_{i}}}$, then

$$
\frac{\partial \varphi_{i}}{\partial x_{i}}(x)=\rho(x)^{-\alpha_{i}}-x_{i} \alpha_{i} \rho(x)^{-\alpha_{i}-1} \frac{\partial \rho(x)}{\partial x_{i}} .
$$

By $F(x, \rho)=1$, we get

$$
\frac{\partial \rho(x)}{\partial x_{i}}=\frac{x_{i} \rho(x)^{-2 \alpha_{i}}}{\sum_{j=1}^{n} \alpha_{j} x_{j}^{2} \rho(x)^{-2 \alpha_{j}-1}} .
$$

This together with (3.2) shows that

$$
\begin{aligned}
\frac{\partial \varphi_{i}}{\partial x_{i}}(x) & =\rho(x)^{-\alpha_{i}}-x_{i} \alpha_{i} \rho(x)^{-\alpha_{i}-1} \frac{x_{i} \rho(x)^{-2 \alpha_{i}}}{\sum_{j=1}^{n} \alpha_{j} x_{j}^{2} \rho(x)^{-2 \alpha_{j}-1}} \\
& =\rho(x)^{-\alpha_{i}}\left(1-\frac{\alpha_{i} x_{i}^{2} \rho(x)^{-2 \alpha_{i}}}{\sum_{j=1}^{n} \alpha_{j} x_{j}^{2} \rho(x)^{-2 \alpha_{j}}}\right) .
\end{aligned}
$$


Since $\frac{\left|x_{i}\right|}{\rho(x)^{\alpha_{i}}} \leq 1$ and

$$
\min \left\{\alpha_{i}\right\} \leq \sum_{j=1}^{n} \alpha_{j} x_{j}^{2} \rho(x)^{-2 \alpha_{j}} \leq \max \left\{\alpha_{i}\right\},
$$

we get by (3.3) and (3.4)

$$
\left|\frac{\partial \varphi_{i}}{\partial x_{i}}(x)\right| \leq\left(1+\frac{\alpha_{i}}{\min \left\{\alpha_{j}\right\}}\right) \rho(x)^{-\alpha_{i}} .
$$

Applying (3.5) and the mean value theorem to $\varphi_{i}(x)$, we have

$$
\left|\frac{x_{i}-y_{i}}{\rho(x-y)^{\alpha_{i}}}-\frac{x_{i}}{\rho(x)^{\alpha_{i}}}\right| \leq\left|\frac{\partial \varphi_{i}}{\partial x_{i}}(\xi)\right|\left|y_{i}\right| \leq\left(1+\alpha_{i}\right) \rho(\xi)^{-\alpha_{i}}\left|y_{i}\right|,
$$

where $\xi=t(x-y)+(1-t) x$ for some $t \in(0,1)$. Then we have

$$
\rho(x) \leq \rho(\xi)+\rho(x-\xi)=\rho(\xi)+\rho(t y) \leq \rho(\xi)+\rho(y) .
$$

Bearing in mind $\rho(x) \geq 4 \rho(y)$, so $\rho(\xi) \geq 3 \rho(y)$ and $\rho(\xi) \geq 3 / 4 \rho(x)$. This together (3.6) yields

$$
\begin{aligned}
\left|\frac{x_{i}-y_{i}}{\rho(x-y)^{\alpha_{i}}}-\frac{x_{i}}{\rho(x)^{\alpha_{i}}}\right| & \leq\left(1+\alpha_{i}\right)(4 / 3)^{\alpha_{i}} \rho(x)^{-\alpha_{i}}\left|y_{i}\right| \\
& \leq 2 \alpha_{i}(4 / 3)^{\alpha_{i}} \rho(x)^{-\alpha_{i}}\left|y_{i}\right| .
\end{aligned}
$$

Notice that $\rho\left(\delta_{\mu} x\right)=\mu \rho(x)$ for $\mu>0$, it follows from (3.1) and (3.7)

$$
\begin{aligned}
\rho\left((x-y)^{\prime}-x^{\prime}\right) & \leq \rho\left(2 \alpha_{1}\left(\frac{4}{3 \rho(x)}\right)^{\alpha_{1}}\left|y_{1}\right|, \ldots, 2 \alpha_{n}\left(\frac{4}{3 \rho(x)}\right)^{\alpha_{n}}\left|y_{n}\right|\right) \\
& \leq \frac{4}{3} \rho(x)^{-1} \max \left\{\left(2 \alpha_{i}\right)^{1 / \alpha_{i}}\right\} \rho\left(\left|y_{1}\right|, \ldots,\left|y_{n}\right|\right) \\
& =\frac{4}{3} \rho(x)^{-1} \max \left\{\left(2 \alpha_{i}\right)^{1 / \alpha_{i}}\right\} \rho(y) \\
& \leq 3 \rho(x)^{-1} \rho(y) .
\end{aligned}
$$

Lemma 3.2. If $\Omega$ satisfies the conditions (1.4) and (1.8), then for $\rho(x) \geq$ $4 \rho(y)$

$$
\left|\frac{\Omega(x-y)}{\rho(x-y)^{\alpha}}-\frac{\Omega(x)}{\rho(x)^{\alpha}}\right| \leq \frac{C}{\rho(x)^{\alpha}\left(\log \frac{\rho(x)}{\rho(y)}\right)^{\gamma}} .
$$


Proof. Since $\rho(x) \geq 4 \rho(y)$, then by (1.4), (1.8) and Lemma 3.1 we get

$$
|\Omega(x-y)-\Omega(x)| \leq \frac{C}{\left(\log \frac{\rho(x)}{\rho(y)}\right)^{\gamma}} .
$$

Now we claim that

$$
\left|\rho(x)^{-\alpha}-\rho(x-y)^{-\alpha}\right| \leq C \frac{\rho(y)}{\rho(x)^{\alpha+1}} .
$$

In fact, we know $3 / 4 \leq \frac{\rho(x-y)}{\rho(x)} \leq 5 / 4$ by $\rho(x) \geq 4 \rho(y)$. On the other hand, using the convexity of $t^{-\alpha}$, it is easy to check that

$$
\frac{1-(1 / 2)^{-\alpha}}{1-1 / 2} \leq \frac{1-t^{-\alpha}}{1-t} \quad \text { for } \quad t>1 / 2 \quad \text { and } \quad t \neq 1 .
$$

Hence

$$
\left|1-t^{-\alpha}\right| \leq 2\left(2^{\alpha}-1\right)|1-t| \quad \text { for } t>1 / 2 .
$$

Thus, for $\rho(x) \geq 4 \rho(y)$ we have

$$
\left|\rho(x)^{-\alpha}-\rho(x-y)^{-\alpha}\right| \leq 2\left(2^{\alpha}-1\right) \frac{\rho(y)}{\rho(x)^{\alpha+1}} .
$$

Hence we get (3.9). Applying (3.8), (3.9) and notice that $\Omega \in L^{\infty}\left(S^{n-1}\right)$, we have

$$
\begin{aligned}
& \left|\frac{\Omega(x-y)}{\rho(x-y)^{\alpha}}-\frac{\Omega(x)}{\rho(x)^{\alpha}}\right| \\
& \quad \leq \frac{|\Omega(x-y)-\Omega(x)|}{\rho(x)^{\alpha}}+|\Omega(x-y)|\left|\rho(x-y)^{-\alpha}-\rho(x)^{-\alpha}\right| \\
& \quad \leq \frac{C}{\rho(x)^{\alpha}\left(\log \frac{\rho(x)}{\rho(y)}\right)^{\gamma}}+C \frac{\rho(y)}{\rho(x)^{\alpha+1}} \\
& \quad \leq \frac{C}{\rho(x)^{\alpha}\left(\log \frac{\rho(x)}{\rho(y)}\right)^{\gamma}} .
\end{aligned}
$$

Lemma 3.3 ([9]). Let $|E|$ be a set of finite measure. Then

$$
\left\|\chi_{E}\right\|_{L_{\psi^{*}}}=|E| \psi^{-1}\left(|E|^{-1}\right)
$$

Lemma 3.4 ([6]). If $0<\beta \leq 1$ and $\varphi(t)=t^{\beta}$, then $\mathrm{BMO}_{\varphi}\left(\mathrm{R}^{n}, \rho\right)=$ $\Lambda_{\beta}\left(\mathrm{R}^{n}, \rho\right)$. 


\section{Proof of Theorem 1.2}

In the proof of Theorem 1.2, we will use some idea taken from [11]. Suppose that $[b, T]$ is a bounded operator from $L^{p}\left(\mathrm{R}^{n}\right)$ to $L_{\psi}\left(\mathrm{R}^{n}\right)$, we are going to prove that $b \in \mathrm{BMO}_{\varphi}\left(\mathrm{R}^{n}, \rho\right)$. Below, $B_{j}(j=1, \ldots, 15)$ denotes the positive constant depending only on $\Omega, p, \alpha, \gamma$ and $B_{i}(1 \leq i<j)$.

Without loss of generality, we assume $\|[b, T]\|_{L^{p} \rightarrow L_{\psi}}=1$. We wish to prove that there exists a constant $B:=B(\Omega, p, \alpha, \gamma)$ such that for any $x_{0} \in \mathrm{R}^{n}$ and $r \in \mathbf{R}_{+}$,

$$
N:=\frac{1}{\left|\mathscr{E}\left(x_{0}, r\right)\right| \varphi(r)} \int_{\mathscr{E}\left(x_{0}, r\right)}\left|b(y)-a_{0}\right| d y \leq B,
$$

where $a_{0}=\left|\mathscr{E}\left(x_{0}, r\right)\right|^{-1} \int_{\mathscr{E}\left(x_{0}, r\right)} b(y) d y$. If we denote

$$
\widetilde{T} f(x)=\text { p.v. } \int_{\mathrm{R}^{n}} \frac{\widetilde{\Omega}(x-y)}{\rho(x-y)^{\alpha}} f(y) d y,
$$

where $\widetilde{\Omega}(x)=-\Omega(-\underset{\widetilde{T}}{)}$, then it is easy to see that $[b, \widetilde{T}]$ is the adjoint operator of $[b, T]$. Hence $\|[b, \widetilde{T}]\|_{L_{\psi^{*} \rightarrow L^{p^{\prime}}}}=1$. Notice that $\left[b-a_{0}, \widetilde{T}\right]=[b, \widetilde{T}]$, thus we may assume $a_{0}=0$. Let

$$
f(y)=\left[\operatorname{sgn}(b(y))-c_{0}\right] \chi_{\mathscr{E}\left(x_{0}, r\right)}(y),
$$

where $c_{0}=\frac{1}{\left|\mathscr{E}\left(x_{0}, r\right)\right|} \int_{\mathscr{E}\left(x_{0}, r\right)} \operatorname{sgn}(b(y)) d y$. By $\frac{1}{\left|\mathscr{E}\left(x_{0}, r\right)\right|} \int_{\mathscr{E}\left(x_{0}, r\right)} b(y) d y=a_{0}=$ 0 , it is easy to check that $\left|c_{0}\right|<1$. Moreover, the following properties of $f$ are obvious:

$$
\begin{gathered}
\|f\|_{\infty} \leq 2, \\
\operatorname{supp} f \subset \mathscr{E}\left(x_{0}, r\right), \\
\int_{\mathrm{R}^{n}} f(y) d y=0, \\
f(y) b(y)>0, \\
\frac{1}{\left|\mathscr{E}\left(x_{0}, r\right)\right| \varphi(r)} \int_{\mathrm{R}^{n}} f(y) b(y) d y=N .
\end{gathered}
$$

Since $\Omega$ satisfies (1.5) and (1.8), then there exists a positive number $B_{1}<1$ such that

$$
\sigma(\Lambda)=\sigma\left(\left\{x^{\prime} \in S^{n-1}: \Omega\left(x^{\prime}\right) \geq \frac{2 C_{1}}{\left(\log \frac{2}{B_{1}}\right)^{\gamma}}\right\}\right)>0
$$


where $\sigma$ is the measure on $S^{n-1}$ which is induced from the Lebesgue measure on $\mathrm{R}^{n}$. Let

$$
\Lambda=\left\{x^{\prime} \in S^{n-1}: \Omega\left(x^{\prime}\right) \geq \frac{2 C_{1}}{\left(\log \frac{2}{B_{1}}\right)^{\gamma}}\right\}>0,
$$

then $\Lambda$ is a closed set. If denote $\tilde{\Lambda}=\left\{x^{\prime} \in S_{\widetilde{\Lambda}}^{n-1}:-x^{\prime}=(-x)^{\prime} \in \Lambda\right\}$, then $\sigma(\widetilde{\Lambda})=\sigma(\Lambda)>0$. We claim that if $x^{\prime} \in \widetilde{\Lambda}$ and $y^{\prime} \in S^{n-1}$ satisfying $\rho\left(y^{\prime}-(-x)^{\prime}\right) \leq B_{1}$, then

$$
\Omega\left(y^{\prime}\right) \geq \frac{C_{1}}{\left(\log \frac{2}{B_{1}}\right)^{\gamma}} .
$$

In fact, since

$$
\left|\Omega\left((-x)^{\prime}\right)-\Omega\left(y^{\prime}\right)\right| \leq \frac{C_{1}}{\left(\log \frac{2}{\rho\left(y^{\prime}-(-x)^{\prime}\right)}\right)^{\gamma}} \leq \frac{C_{1}}{\left(\log \frac{2}{B_{1}}\right)^{\gamma}}
$$

and note that $\Omega\left((-x)^{\prime}\right) \geq 2 \frac{C_{1}}{\left(\log \frac{2}{B_{1}}\right)^{\gamma}}$, we have $\Omega\left(y^{\prime}\right) \geq \frac{C_{1}}{\left(\log \frac{2}{B_{1}}\right)^{\gamma}}$. Now denote

$$
G=\left\{x \in \mathrm{R}^{n}: \rho\left(x-x_{0}\right)>B_{2} r \text { and }\left(x-x_{0}\right)^{\prime} \in \tilde{\Lambda}\right\},
$$

where $B_{2}=3 B_{1}^{-1}+1$. Then for $x \in G$,

$$
\begin{aligned}
|[b, \widetilde{T}] f(x)| \\
\quad \geq|\widetilde{T}(b f)(x)|-|b(x)||\widetilde{T} f(x)| \\
\quad=\left|\int_{\mathrm{R}^{n}} \frac{\Omega\left((y-x)^{\prime}\right)}{\rho(y-x)^{\alpha}} b(y) f(y) d y\right|-|b(x)|\left|\int_{\mathrm{R}^{n}} \frac{\Omega\left((y-x)^{\prime}\right)}{\rho(y-x)^{\alpha}} f(y) d y\right| \\
\quad:=I_{1}-I_{2} .
\end{aligned}
$$

We first give the estimate of $I_{1}$. If $\rho\left(y-x_{0}\right)<r$, then

$$
\rho\left(x-x_{0}\right)>B_{2} \rho\left(y-x_{0}\right)>4 \rho\left(y-x_{0}\right) .
$$

By Lemma 3.1, we see that

$$
\rho\left((y-x)^{\prime}-\left(x_{0}-x\right)^{\prime}\right) \leq 3 \frac{\rho\left(y-x_{0}\right)}{\rho\left(x-x_{0}\right)} \leq B_{1} .
$$

Then $\Omega\left((y-x)^{\prime}\right) \geq \frac{C_{1}}{\left(\log \frac{2}{B_{1}}\right)^{\gamma}}$ by (4.9). Thus, it follows from (4.4), (4.6) and 
(4.7) that

$$
\begin{aligned}
I_{1} & \geq \frac{C_{1}}{\left(\log \frac{2}{B_{1}}\right)^{\gamma}} \int_{\mathscr{E}\left(x_{0}, r\right)} b(y) f(y) \rho(y-x)^{-\alpha} d y \\
& \geq \frac{C_{1}}{\left(\log \frac{2}{B_{1}}\right)^{\gamma}} \int_{\mathscr{E}\left(x_{0}, r\right)} b(y) f(y)\left(\rho\left(x-x_{0}\right)+\rho\left(y-x_{0}\right)\right)^{-\alpha} d y \\
& \geq \frac{C_{1}}{\left(\log \frac{2}{B_{1}}\right)^{\gamma}} 2^{-\alpha} \int_{\mathscr{E}\left(x_{0}, r\right)} b(y) f(y) \rho\left(x-x_{0}\right)^{-\alpha} d y \\
& =B_{3} r^{\alpha} \varphi(r) N \rho\left(x-x_{0}\right)^{-\alpha} .
\end{aligned}
$$

Let us turn to $I_{2}$. Since $\rho\left(x-x_{0}\right) \geq 4 \rho\left(y-x_{0}\right)$, by (4.3), (4.4), (4.5) and Lemma 3.2 we get

$$
\begin{aligned}
I_{2} & \leq|b(x)| \int_{\mathscr{E}\left(x_{0}, r\right)}|f(y)|\left|\frac{\Omega(y-x)}{\rho(y-x)^{\alpha}}-\frac{\Omega\left(x_{0}-x\right)}{\rho\left(x_{0}-x\right)^{\alpha}}\right| d y \\
& \leq C|b(x)| \int_{\mathscr{E}\left(x_{0}, r\right)} \frac{|f(y)|}{\rho\left(x-x_{0}\right)^{\alpha}\left(\log \frac{\rho\left(x-x_{0}\right)}{r}\right)^{\gamma}} d y \\
& \leq B_{4} r^{\alpha}|b(x)| \rho\left(x-x_{0}\right)^{-\alpha}\left(\log \frac{\rho\left(x-x_{0}\right)}{r}\right)^{-\gamma} .
\end{aligned}
$$

Set

$$
\begin{aligned}
F=\left\{x \in G:|b(x)|>\frac{B_{3} N \varphi(r)}{2 B_{4}}\left(\log \frac{\rho\left(x-x_{0}\right)}{r}\right)^{\gamma}\right. \\
\text { and } \left.\rho\left(x-x_{0}\right)<N^{p / \alpha} r\right\} .
\end{aligned}
$$

By (4.10), (4.11) and (4.12), we have

$$
\begin{aligned}
& \|f\|_{L_{\psi^{*}}}^{p^{\prime}} \\
& \quad \geq \int_{\mathrm{R}^{n}}|[b, \widetilde{T}] f(x)|^{p^{\prime}} d x \\
& \quad \geq \int_{(G \backslash F) \cap\left\{\rho\left(x-x_{0}\right)<N^{p / \alpha} r\right\}}\left(\frac{1}{2} B_{3} N \varphi(r) r^{\alpha} \rho\left(x-x_{0}\right)^{-\alpha}\right)^{p^{\prime}} d x \\
& \quad \geq \int_{\left\{B_{5}\left(|F|+\left(B_{2} r\right)^{\alpha}\right)^{1 / \alpha}<\rho\left(x-x_{0}\right)<N^{p / \alpha} r\right\} \cap G}\left(\frac{1}{2} B_{3} N \varphi(r) r^{\alpha} \rho\left(x-x_{0}\right)^{-\alpha}\right)^{p^{\prime}} d x
\end{aligned}
$$




$$
\begin{aligned}
& =\left(\frac{B_{3} N \varphi(r) r^{\alpha}}{2}\right)^{p^{\prime}} \int_{B_{5}\left(|F|+\left(B_{2} r\right)^{\alpha}\right)^{1 / \alpha}}^{N^{p / \alpha} r} t^{-\alpha p^{\prime}+\alpha-1} d t \int_{\widetilde{\Lambda}} J\left(x^{\prime}\right) d \sigma\left(x^{\prime}\right) \\
& \geq \sigma(\widetilde{\Lambda}) \frac{\left(\frac{B_{3} N \varphi(r) r^{\alpha}}{2}\right)^{p^{\prime}}}{\alpha-\alpha p^{\prime}}\left[N^{p\left(1-p^{\prime}\right)} r^{\alpha\left(1-p^{\prime}\right)}-B_{5}^{\left(1-p^{\prime}\right) \alpha}\left(|F|+\left(B_{2} r\right)^{\alpha}\right)^{1-p^{\prime}}\right] .
\end{aligned}
$$

Since $-p^{\prime}=p\left(1-p^{\prime}\right)$, thus we have

$$
\begin{aligned}
\left(|F|+\left(B_{2} r\right)^{\alpha}\right)^{1-p^{\prime}} & \\
& \leq B_{6} N^{p\left(1-p^{\prime}\right)}\left(r^{\alpha\left(1-p^{\prime}\right)}+\varphi(r)^{p\left(1-p^{\prime}\right)} r^{p\left(1-p^{\prime}\right) \alpha}\|f\|_{L^{\psi^{*}}}^{p\left(p^{\prime}-1\right)}\right)
\end{aligned}
$$

By Lemma 3.3 and the definition of $f$, we have $\|f\|_{L_{\psi^{*}}} \leq C r^{\alpha} \psi^{-1}\left(r^{-\alpha}\right)=$ $C \varphi(r) r^{\frac{\alpha}{p^{\prime}}}$. Hence $|F|+\left(B_{2} r\right)^{\alpha} \geq B_{7} N^{p} r^{\alpha}$.

If $N \leq\left(2 B_{7}^{-1} B_{2}^{\alpha}\right)^{1 / p}$, then Theorem 1.2 is proved. If $N>\left(2 B_{7}^{-1} B_{2}^{\alpha}\right)^{1 / p}$, we get

$$
|F| \geq \frac{B_{7}}{2} N^{p} r^{\alpha}
$$

On the other hand, for $x \in \mathscr{E}\left(x_{0}, r\right)$ and $y \in F$ we have the following facts:

(a) $\rho(x-y) \sim \rho\left(y-x_{0}\right)$;

(b) $\Omega\left((x-y)^{\prime}\right) \geq \frac{C_{1}}{\left(\log \frac{2}{B_{1}}\right)^{\gamma}}$.

The fact (a) follows from $F \subset G$ and $B_{2}>4$. By $\rho\left(y-x_{0}\right)>B_{2} \rho\left(x-x_{0}\right)$ and Lemma 3.1, we have $\rho\left((x-y)^{\prime}-\left(x_{0}-y\right)^{\prime}\right) \leq B_{1}$ and then get (b) by applying (4.9).

Now, we denote $g(y)=(\operatorname{sgn}(b(y))) \chi_{F}(y)$. For $x \in \mathscr{E}\left(x_{0}, r\right)$ we have

$$
\begin{aligned}
\mid[b, & T] g(x) \mid \\
& \geq|T(b g)(x)|-|b(x)||T g(x)| \\
& \geq \int_{F} \frac{\Omega\left((x-y)^{\prime}\right)}{\rho(x-y)^{\alpha}}|b(y)| d y-|b(x)| \int_{F} \frac{\left|\Omega\left((x-y)^{\prime}\right)\right|}{\rho(x-y)^{\alpha}}|g(y)| d y \\
& =K_{1}-K_{2} .
\end{aligned}
$$

We first estimate $K_{2}$. Since $\Omega \in L^{\infty}\left(S^{n-1}\right)$ and $\rho(x-y) \sim \rho\left(y-x_{0}\right)$, we 
have

$$
\begin{aligned}
K_{2} & \leq B_{8}|b(x)| \int_{F} \rho\left(y-x_{0}\right)^{-\alpha} d y \\
& \leq B_{8}|b(x)| \int_{B_{2} r}^{N^{p / \alpha} r} \rho^{-1} d \rho \int_{S^{n-1}} J\left(y^{\prime}\right) d \sigma\left(y^{\prime}\right) \\
& \leq B_{9}|b(x)| \log N .
\end{aligned}
$$

Now regarding $K_{1}$. By the facts (a) and (b), we have

$$
K_{1} \geq \frac{C_{1}}{\left(\log \frac{1}{B_{1}}\right)^{\gamma}} \frac{B_{3} N \varphi(r)}{2 B_{4}} \int_{F} \rho\left(y-x_{0}\right)^{-\alpha}\left(\log \frac{\rho\left(y-x_{0}\right)}{r}\right)^{\gamma} d y .
$$

The estimate of $K_{1}$ is divided into two cases, namely

$$
1^{\circ}: \quad \gamma \geq \alpha ; \quad 2^{\circ}: 1<\gamma<\alpha
$$

Case $1^{\circ}$. Since the function $s \rightarrow \frac{\log s}{s}$ is decreasing for $s \geq 3$ and $4 r<B_{2} r<$ $\rho(y)<N^{p / \alpha} r$ for $y \in F$, hence by (4.16) and (4.13) we get

$$
\begin{aligned}
K_{1} & \geq B_{10} N \varphi(r) r^{-\alpha} \int_{F}\left(\frac{\log \frac{\rho\left(y-x_{0}\right)}{r}}{\frac{\rho\left(y-x_{0}\right)}{r}}\right)^{\alpha}\left(\log \frac{\rho\left(y-x_{0}\right)}{r}\right)^{\gamma-\alpha} d y \\
& \geq B_{10} N \varphi(r)\left(\log B_{2}\right)^{\gamma-\alpha}\left(\frac{\log N^{p / \alpha}}{N^{p / \alpha}}\right)^{\alpha} \frac{B_{7}}{2} N^{p} \\
& =B_{11} \varphi(r) N(\log N)^{\alpha} .
\end{aligned}
$$

Case $2^{\circ}$. In this case, the function $s \rightarrow \frac{(\log s)^{\gamma}}{s^{\alpha}}$ is still decreasing for $s \geq 3$. By $4 r<B_{2} r<\rho\left(y-x_{0}\right)<N^{p / \alpha} r$ if $y \in F$ and (4.16), (4.13) we have

$$
\begin{aligned}
K_{1} & \geq B_{10} N \varphi(r) r^{-\alpha} \int_{F} \frac{\left(\log \frac{\rho\left(y-x_{0}\right)}{r}\right)^{\gamma}}{\left(\frac{\rho\left(y-x_{0}\right)}{r}\right)^{\alpha}} d y \\
& \geq B_{10} \frac{B_{7}}{2} \varphi(r) N^{p} N \frac{\left(\log N^{p / \alpha}\right)^{\gamma}}{N^{p}} \\
& =B_{12} \varphi(r) N(\log N)^{\gamma} .
\end{aligned}
$$

Summing up the estimates in Case $1^{\circ}$ and Case $2^{\circ}$, there exists a constant $\tau>1$ such that

$$
K_{1} \geq B_{13} \varphi(r) N(\log N)^{\tau}
$$


Thus, by (4.14), (4.15) and (4.17), we get

(4.18) $|[b, T] g(x)| \geq B_{13} \varphi(r) N(\log N)^{\tau}-B_{9}|b(x)| \log N, \quad x \in \mathscr{E}\left(x_{0}, r\right)$.

Since $\|[b, T]\|_{L^{p} \rightarrow L_{\psi}}=1$. Thus by (4.13), (4.18) and the generalized Hölder's inequality (1.3), we have

$$
\begin{aligned}
B_{14} r^{\frac{\alpha}{p}+\alpha} \psi^{-1}\left(r^{-\alpha}\right) N \\
\quad \geq r^{\alpha} \psi^{-1}\left(r^{-\alpha}\right)\left(\int_{\rho\left(x-x_{0}\right) \leq N^{\frac{p}{\alpha}} r} d x\right)^{1 / p} \\
\quad \geq r^{\alpha} \psi^{-1}\left(r^{-\alpha}\right)\left(\int_{F} d x\right)^{1 / p} \\
\quad=r^{\alpha} \psi^{-1}\left(r^{-\alpha}\right)\|g\|_{p} \\
\geq\left\|\chi_{\mathscr{E}\left(x_{0}, r\right)}\right\|_{L_{\psi^{*}}}\|[b, T] g\|_{L_{\psi}} \\
\geq \int_{\mathscr{E}\left(x_{0}, r\right)}|[b, T] g(x)| d x \\
\geq B_{13} \varphi(r) N(\log N)^{\tau} \int_{\mathscr{E}\left(x_{0}, r\right)} d x-B_{9} \log N \int_{\mathscr{E}\left(x_{0}, r\right)}|b(x)| d x \\
\geq B_{13} \varphi(r) r^{\alpha} N(\log N)^{\tau}-B_{9} \varphi(r) r^{\alpha} N \log N .
\end{aligned}
$$

This shows that

$$
B_{14} r^{\frac{\alpha}{p}} \psi^{-1}\left(r^{-\alpha}\right) \geq B_{13}(\log N)^{\tau} \varphi(r)-B_{9} \log N \varphi(r) .
$$

Since $\varphi(r)=r^{\frac{\alpha}{p}} \psi^{-1}\left(r^{-\alpha}\right)$, then (4.19) becomes $B_{14} \geq B_{13}(\log N)^{\tau}-$ $B_{9} \log N$. Therefore, there exists a constant $B:=B(\Omega, p, \alpha, \gamma)$ such that $N \leq B$. Thus we finish the proof of Theorem 1.2.

ACKNOWLEDGEMENT. The authors would like to express their deep gratitude to the referee for his/her reading carefully and valuable comments.

\section{REFERENCES}

1. Bramanti, M., and Cerutti, M., Commutators of singular integrals on homogeneous spaces, Boll Un. Mat. Ital. B (7) 10 (1996), 843-883.

2. Coifman, R., Rochberg, R., and Weiss, G., Factorization theorems for Hardy spaces in several variables, Ann. of Math. (2) 103 (1976), 611-636.

3. Chen, Y., and Ding, Y., $L^{p}$ bounds for the commutator of parabolic singular integral with rough kernels, Potential Anal. 27 (2007), 313-334. 
4. Chen, Y., Ding, Y., and Fan, D., A parabolic singular integral operator with rough kernel, J. Aust. Math. Soc. 84 (2008), 163-179.

5. Fabes, E., and Rivière, N., Singular integrals with mixed homogeneity, Studia Math. 27 (1966), 19-38.

6. Janson, S., Mean oscillation and commutators of singular integral operators, Ark. Mat. 16 (1978), 263-270.

7. Nagel, A., Rivière, N., and Wainger, S., On Hilbert transforms along curves II, Amer. J. Math. 98 (1976), 395-403.

8. Palagachev, D., and Softova, L., Singular integral operators, Morrey spaces and fine regularity of solutions to PDE's, Potential Anal. 20 (2004), 237-263.

9. Rao, M., and Ren, Z., Theorey of Orlicz Spaces, Monogr. Textbooks Pure Appl. Math. 146, Marcel Dekker, New York 1991.

10. Stein, E. M., Singular Integrals and Differentiability Properties of Functions, Princeton Math. Series 30, Princeton Univ. Press, Princeton, NJ 1970.

11. Uchiyama, A., On the compactness of operators of Hankel type, Tôhoku Math. J. 30 (1978), $163-171$.

YANPING CHEN

APPLIED SCIENCE SCHOOL

UNIVERSITY OF SCIENCE AND TECHNOLOGY BEIJING

BEIJING 100083

CHINA

E-mail: yanpingch@126.com
YONG DING (Corresponding author) SCHOOL OF MATHEMATICAL SCIENCES BEIJING NORMAL UNIVERSITY LABORATORY OF MATHEMATICS AND COMPLEX SYSTEMS (BNU) MINISTRY OF EDUCATION BEIJING 100875

CHINA

E-mail: dingy@bnu.edu.cn 